\title{
How dieting might make some fatter: modeling weight cycling toward obesity from a perspective of body composition autoregulation
}

\author{
Philippe Jacquet $^{1,2} \cdot$ Yves Schutz $^{1} \cdot$ Jean-Pierre Montani ${ }^{1} \cdot$ Abdul Dulloo $^{1}$
}

Received: 20 August 2019 / Revised: 9 January 2020 / Accepted: 6 February 2020 / Published online: 25 February 2020

(c) The Author(s) 2020.

\begin{abstract}
The notion that dieting makes some people fatter has in the past decade gained considerable interest from both epidemiological predictions and biological plausibility. Several large-scale prospective studies have suggested that dieting to lose weight is associated with future weight gain and obesity, with such predictions being stronger and more consistent among dieters who are in the normal range of body weight rather than in those with obesity. Furthermore, the biological plausibility that dieting predisposes people who are lean (rather than those with overweight or obesity) to regain more body fat than what had been lost (referred to as fat overshooting) has recently gained support from a re-analysis of data on body composition during weight loss and subsequent weight recovery from the classic longitudinal Minnesota Starvation Experiment. These have revealed an inverse exponential relationship between the amount of fat overshot and initial adiposity, and have suggested that a temporal desynchronization in the recoveries of fat and lean tissues, in turn residing in differences in lean-fat partitioning during weight loss vs. during weight recovery (with fat recovery faster than lean tissue recovery) is a cardinal feature of fat overshooting. Within a conceptual framework that integrates the relationship between post-dieting fat overshooting with initial adiposity, the extent of weight loss and the differential lean-fat partitioning during weight loss vs. weight recovery, we describe here a mathematical model of weight cycling to predict the excess fat that could be gained through repeated dieting and multiple weight cycles from a standpoint of body composition autoregulation.
\end{abstract}

\section{Background}

In parallel to the increasing prevalence of overweight and obesity worldwide, the prevalence of dieting is also rising, and current estimates indicate that $40 \%$ of adults have tried

Supplementary information The online version of this article (https:// doi.org/10.1038/s41366-020-0547-1) contains supplementary material, which is available to authorized users.

Philippe Jacquet

philippe.jacquet@unil.ch

$\triangle$ Abdul Dulloo

abdul.dulloo@unifr.ch

1 Department of Endocrinology, Metabolism \& Cardiovascular system, Faculty of Science \& Medicine, University of Fribourg, Fribourg, Switzerland

2 Scientific Computing and Research Support Unit, Computer Center, University of Lausanne, Lausanne, Switzerland to lose weight at some point during the last 5 years [1]. With studies of the long-term outcomes showing that at least onethird of dieters regain more weight than they lost [2], together with prospective studies indicating that dietingwhether in adults [3-11], adolescents [12-16] or children [17-19] — predicts future weight gain and obesity, there is concern as to whether dieting may paradoxically be promoting exactly the opposite of what it is intended to achieve [20-22].

Indeed, the notion that dieting to lose weight is counterproductive for weight management in that people may regain more fat than they lose through each cycle of weight loss/regain was embodied in the title of a book published in 1983: 'Dieting makes you fat' [23]. This notion remains controversial and the subject of frequent debates among scientists [24-31] despite the conclusion of a US National Task Force on the Prevention and Treatment of Obesity [32] that to quote: 'the available evidence is not sufficiently compelling to override the potential benefits of moderate weight loss in significantly obese patients'. 
While this conclusion made 25 years ago may still be valid today, its specification pertaining to patients with obesity was perhaps a premonition for the subsequent findings from several prospective studies suggesting that it was dieting in people of normal body weight, rather than in those with overweight or obesity, that was most strongly and consistently associated with future weight gain $[33,34]$ and risks for cardiometabolic diseases [34]. In particular, in a 6-15-year follow-up study in young and middle-aged population groups, the risk of major weight gain exceeding $10 \mathrm{~kg}$ was found to be higher (by twofold) in dieters than in non-dieters among those initially of normal body weight but not among those initially with overweight or obesity [6]. In another large populationbased cohort with a 10-year follow-up from adolescence to young adulthood [16], a dose-dependent association was found between the frequency of intentional weight loss episodes of more than $5 \mathrm{~kg}$ and the gain in body mass index (BMI) over the 10-year follow-up, as well as with the risk of overweight at 25 years of age. Furthermore, those in the lowest BMI category at baseline gained more weight than those in the intermediate or high baseline BMI category [16]. More recently, in a study based on a representative adult population sample (Finnish Health 2000 Survey) and on its follow-up examination 11 years later in 2,785 adults aged 30-69, the increases in BMI and waist circumference were found to be greater in dieters than in non-dieters, but notably greatest in dieters who reported that they had lost weight or experienced weight fluctuation during the previous year, and in dieters with initially normal weight [11].

Taken together, these studies reinforce the contention that dieting to lose weight and weight cycling most strongly predict future weight gain in those who are lean than in those who are with overweight/obesity. By extension, they reinforce the plausibility that at least in the people without obesity, each cycle of weight loss/regain is accompanied by a greater gain of body fat than is lost. Such a phenomenon referred to as weight (or fat) overshooting is directly supported by the classic longitudinal study of semistarvation and refeeding — the Minnesota Experiment [35]—conducted in young men in the normal range of BMI. During their nutritional rehabilitation after losing 25-29\% of their body weight over 24 weeks of semistarvation, they showed fat overshooting of $4 \mathrm{~kg}$ on average but ranging between 0 and $9 \mathrm{~kg}$, with those showing higher fat overshooting being the leanest, as evidenced from an inverse exponential relationship between the $\mathrm{kg}$ of fat overshot and initial (pre-starvation) body fat $\%$ [36]. Thus, a high dependency of postdieting fat overshooting upon the initial adiposity is a central tenet in explaining the findings of prospective studies showing a more consistent association between dieting to lose weight and increased risks for major weight gain in individuals initially of normal-weight than in people initially with overweight or obesity $[6,11,16]$.

Against this background, we describe here the development and application of a mathematical model to predict the amount of fat overshoot through multiple weight cycles in pathways from leanness to fatness-albeit from a standpoint of body composition autoregulation.

\section{Development of the model}

\section{Basic concepts}

Several mathematical models have been developed to study the regulation of body weight and body composition in which the initial body composition is a simple function that determined the fraction of energy imbalance partitioned toward deposition or mobilization of body protein vs. fat [37-42]. The model presented here, however, rests upon the notion that the initial body composition could also be a factor in the mechanisms by which weight cycling might predispose people to increased fatness. The basic concepts here underlying this modeling of weight cycling from leanness to fatness rests upon several findings from our previous re-analysis of data from the Minnesota Experiment on changes in body composition, energy intake and basal metabolic rate in the 32 men who completed the 24 weeks of semistarvation and 12 weeks of controlled refeeding, as well as in the 12 subjects who also completed the subsequent 8 weeks of refeeding with ad libitum access to food. These are summarized below:

(i) During weight loss in response to semistarvation, an intrinsic lean-fat partitioning characteristic of the individual (Pss) dictates the relative proportion of body energy derived from fat-free mass (FFM), and that this characteristic, which is conserved during refeeding, is a function of the initial body fat $\%$ $[43,44]$. This is consistent with the theoretical equation developed earlier by Forbes [41] that quantified the non-linear relationship between the fat-free proportion of modest weight changes as a function of the initial body fat, and later extended by Hall [42] to account for the magnitude of body weight changes.

(ii) An adaptive suppression of thermogenesis, which operates to conserve energy during weight loss, persists as a function of fat depletion during weight recovery and serves to accelerate specifically the recovery of fat mass but not that of FFM [43, 45].

(iii) The hyperphagia during ad libitum refeeding is driven not only by the degree of fat depletion, but also by the degree of FFM depletion [46]. 
(iv) The operation of these above-mentioned control systems during refeeding is that body fat recovery reaches completion (to baseline pre-starvation levels) before full recovery of FFM, and that hyperphagia (which is partly driven by FFM depletion) persists until complete FFM recovery, with concomitant accumulation of excess fat and hence fat overshoot [46]. In other words, because of the temporal desynchronization in the complete recovery of fat and FFM, fat overshoot is a prerequisite that enables the recovery of FFM driven by hyperphagia to be completed - a process that is referred to as collateral fattening $[47,48]$. In turn, it can be hypothesized that the temporal desynchronization between completion in fat and FFM recoveries reside in differences in lean-fat partitioning during weight loss vs. during weight recovery.

\section{Mathematical modeling of fat overshoot}

On the basis of the above basic concepts derived from the re-analysis of data from the Minnesota Experiment, we start the modeling of fat overshooting by depicting in Fig. 1 the simulation of changes in body weight and body composition of a subject of normal body weight who goes through the two successive phases of a weight cycle. In the first phase (time 0-1), the subject loses weight through semistarvation (SS), and in the second phase (time 1-2), the subject regains weight through refeeding (RF) until complete recovery of FFM, namely until $F F M_{2}=F F M_{0}$. It is to be noted that (i) it is assumed that at time 0,1 and 2 the body fat (FAT) and fat-free mass (FFM) are known, and (ii) the lines in Fig. 1 are only added to 'guide the eyes', and no assumption is made that the weight loss or gain is linear in time. The body weight of the subject across time is then defined as: $W_{\text {time }}=F A T_{\text {time }}+F F M_{\text {time }}$, with time $=0$, 1 or 2 .

As shown in Fig. 1, the subject has an initial weight $W_{0}=F A T_{0}+F F M_{0}$, where $F A T_{0}$ and $F F M_{0}$ are the subject's initial FAT and FFM contents, respectively. Between time 0 and 1 , the subject loses weight $\Delta W_{\mathrm{SS}}>0$ during semistarvation, and reaches at time 1 at the weight given as:

$$
W_{1}=W_{0}-\Delta W_{\mathrm{SS}}=F A T_{1}+F F M_{1},
$$

where $F A T_{1}$ and $F F M_{1}$ are his FAT and FFM contents at time 1 , respectively.

The weight loss may be written as $\Delta W_{\mathrm{SS}}=$ $\triangle F A T_{\mathrm{SS}}+\triangle F F M_{\mathrm{SS}}$, where $\triangle F A T_{\mathrm{SS}}$ and $\triangle F F M_{\mathrm{SS}}$ are the FAT and FFM, respectively, that are lost during the semistarvation process. These quantities may be computed as $\Delta F A T_{\mathrm{SS}}=F A T_{0}-F A T_{1}$ and $\triangle F F M_{\mathrm{SS}}=F F M_{0}-F F M_{1}$. Furthermore, the lean-fat partitioning ratio $P_{\mathrm{SS}}^{\mathrm{m}}$ during the semistarvation phase is defined as the fraction of the weight
Fig. 1 Changes in body weight (W), body fat (FAT) and fatfree mass (FFM) in response to semistarvation (SS) and subsequent refeeding (RF); time 0,1 and 2 represent 'prior to semistarvation', at the 'end of the semistarvation' period, and at the end of the refeeding period when body weight has been completely recovered, respectively. The dynamics of body composition recovery are depicted with FAT and FFM recoveries being desynchronized and resulting in fat overshooting (upper panel) or synchronized so as to reach $100 \%$ recoveries at the same time without fat overshooting (lower panel). All values are expressed as a difference from the corresponding values during the control (time 0) period. More precisely, the values are defined as $F A T_{\text {time }}-F A T_{0}$ for FAT (red circle, dotted line), $F F M_{\text {time }}-$ $F F M_{0}$ for FFM (blue triangle, dashed line) and $W_{\text {time }}-W_{0}$ for $\mathrm{W}$ (black square, solid line).
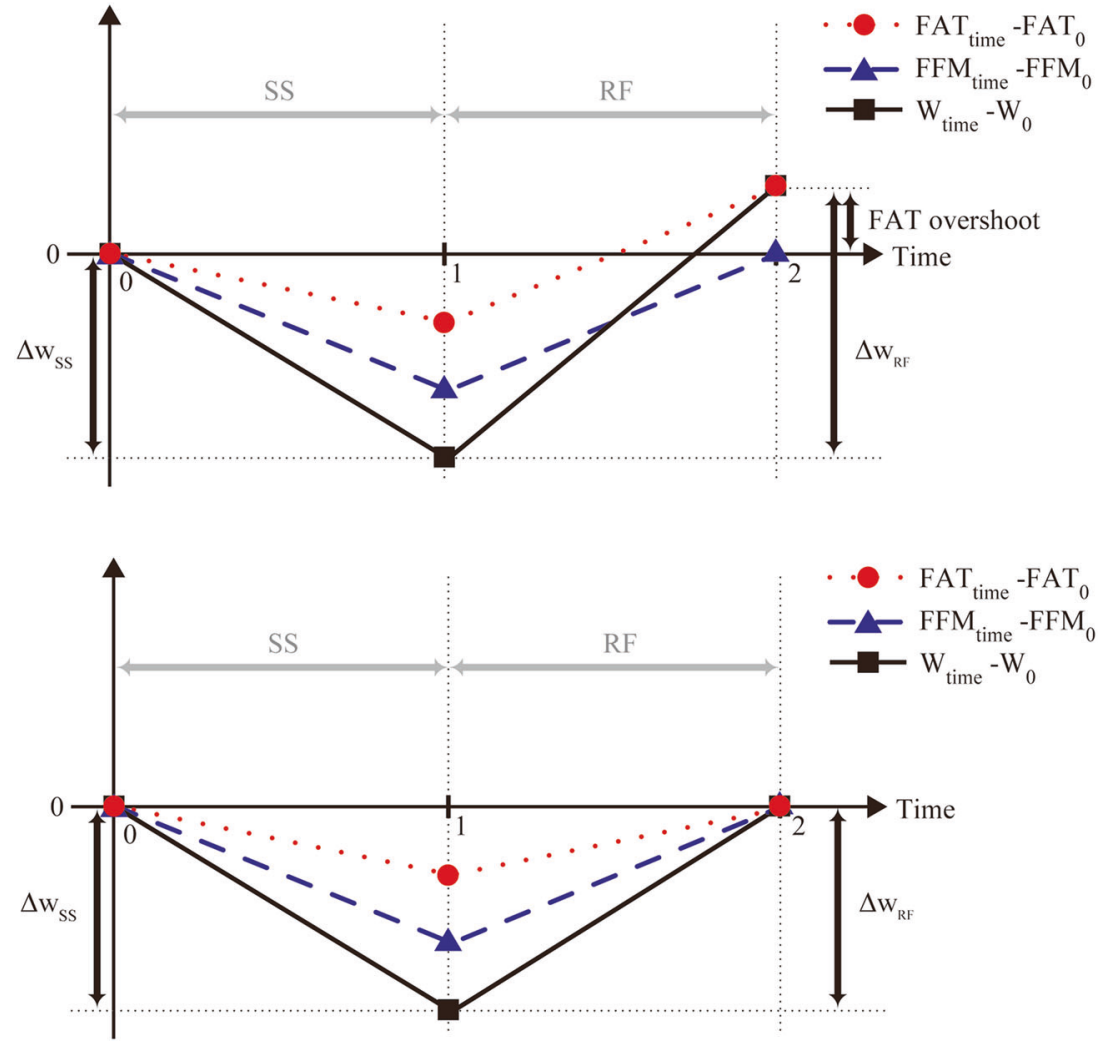
loss as FFM, that is $\Delta F F M_{\mathrm{SS}}=P_{\mathrm{SS}}^{\mathrm{m}} \cdot \Delta W_{\mathrm{SS}}$. Thus, $P_{\mathrm{SS}}^{\mathrm{m}}$ can be written as:

$$
P_{\mathrm{SS}}^{\mathrm{m}}=\frac{1}{1+\frac{\Delta F A T_{\mathrm{SS}}}{\Delta F F M_{\mathrm{SS}}}}
$$

During the refeeding phase (time 1-2) presented in Fig. 1, the subject goes through a weight regain process until his FFM is completely recovered, that is until $F F M_{2}=F F M_{0}$. The weight regained during refeeding can be written as $\Delta W_{\mathrm{RF}}>0$, such that $W_{2}=W_{1}+\Delta W_{\mathrm{RF}}$. As in the semistarvation phase, one may write $\Delta W_{\mathrm{RF}}=\Delta F A T_{\mathrm{RF}}+\Delta F F M_{\mathrm{RF}}$, where $\Delta F A T_{\mathrm{RF}}=F A T_{2}-F A T_{1}$ and $\Delta F F M_{\mathrm{RF}}=F F M_{2}-F F M_{1}$, and introduce the refeeding lean-fat partition ratio $P_{\mathrm{RF}}^{\mathrm{m}}$ as the fraction of the weight regained as FFM, that is $\Delta F F M_{\mathrm{RF}}=P_{\mathrm{RF}}^{\mathrm{m}} \cdot \Delta W_{\mathrm{RF}}$. Thus, $P_{\mathrm{RF}}^{\mathrm{m}}$ can be written as:

$P_{\mathrm{R} F}^{\mathrm{m}}=\frac{1}{1+\frac{\Delta F A T_{\mathrm{R} F}}{\Delta F F M_{\mathrm{R} F}}}$.

Using the equations above, $\Delta W_{\mathrm{RF}}$ can be written as follows:

$$
\begin{aligned}
\Delta W_{\mathrm{RF}} & =\frac{\Delta F F M_{\mathrm{RF}}}{P_{\mathrm{RF}}^{\mathrm{m}}} \\
& =\frac{F F M_{2}-F F M_{1}}{P_{\mathrm{RF}}^{\mathrm{m}}} \\
& =\frac{F F M_{0}-F F M_{1}}{P_{\mathrm{RF}}^{\mathrm{m}}} \\
& =\frac{\Delta F F M_{\mathrm{SS}}}{P_{\mathrm{RF}}^{\mathrm{m}}} \\
& =\frac{P_{\mathrm{SS}}^{\mathrm{m}} \times \Delta W_{\mathrm{SS}}}{P_{\mathrm{RF}}^{\mathrm{m}}} \\
& =\gamma \times \Delta W_{\mathrm{SS}},
\end{aligned}
$$

where

$\gamma=\frac{P_{\mathrm{SS}}^{\mathrm{m}}}{P_{\mathrm{RF}}^{\mathrm{m}}}$

As it will become evident in later sections, the factor $\gamma$, which relates the lean-fat partitioning of the subject during weight loss with that during weight regain, plays a fundamental role in FAT overshooting.

The FAT overshoot corresponds to the excess FAT deposited during the refeeding phase as compared with the initial time 0 , and can be described as follows:

$$
\begin{aligned}
\text { FAT Overshoot } & =F A T_{2}-F A T_{0} \\
& =W_{2}-W_{0} \\
& =W_{1}+\Delta W_{\mathrm{RF}}-W_{0} \\
& =W_{0}-\Delta W_{\mathrm{SS}}+\Delta W_{\mathrm{RF}}-W_{0} \\
& =\Delta W_{\mathrm{RF}}-\Delta W_{\mathrm{SS}} .
\end{aligned}
$$

Thus, using the Eq. (4), we obtain:

$$
\text { FAT Overshoot }=(\gamma-1) \times \Delta W_{\mathrm{SS}} .
$$

This is a key equation in the modeling process. It is an exact mathematical relation without approximation (i.e., not derived from any data) and the parameter $\gamma$ is a cardinal feature in the process leading to fat overshooting. If $\gamma=1$, then the lean-fat partitioning during weight loss is the same as during weight recovery, and both FAT and FFM recoveries reach completion at the same time, i.e., they are synchronized so as to come back exactly to their initial values at the same time, and there is no fat overshooting. By contrast, if $\gamma>1$ (i.e., $P_{\mathrm{RF}}<P_{\mathrm{SS}}$ ), then the quantity $(\gamma-1) \times \Delta W_{\mathrm{SS}}$ is positive, resulting in a temporal desynchronization in complete fat and FFM recoveries, with fat recovery reaching completion before that of FFM recovery, with consequential collateral fattening and fat overshooting. A situation when $\gamma<1$ is in principle possible (e.g., through special diets, very high-intensity exercise during refeeding, or the use of anabolic compounds), but this will not be considered here. As the lean-fat partitioning ratio $\left(P_{\mathrm{SS}}^{\mathrm{m}}\right)$ during semistarvation, which is conserved during refeeding, is to a great extent determined by the initial fat percentage $\%$ $F A T_{0}$ of the subject $[43,44]$, this motivated us to investigate the relationship between $\gamma$ and $\% F A T_{0}$. To this end, we revisited here the Minnesota Experiment with specific focus on the analysis of data on body composition of the 12 men who completed the entire study (i.e., including the ad libitum refeeding phase) and showed varying degrees of fat overshooting, as elaborated step-by-step in the section below.

\section{Revisiting fat overshooting in the Minnesota Experiment}

\section{Step 1. Relationship between lean-fat partitioning during weight loss and initial adiposity}

Before discussing fat overshooting, it is convenient to analyze in more detail the semistarvation mass partition ratio $P_{\mathrm{SS}}^{\mathrm{m}}$ as a function of initial adiposity by combining the two following theoretical constraints:

(i) $\quad P_{\mathrm{SS}}^{\mathrm{m}}=1$ if $\% F A T_{0}=0$, since in this situation all mass (virtually all body energy) must be taken from the FFM compartment when the subject loses weight, and

(ii) $P_{\mathrm{SS}}^{\mathrm{m}}=0$ if $\% F A T_{0}=100$, since in this case no mass (virtually no energy) can be taken from the FFM compartment. 
With an exponential decreasing function of $\% F A T_{0}$, one obtains the following expression:

$P_{\mathrm{SS}}^{\mathrm{m}}=\frac{\left(100-\% F A T_{0}\right)}{100} \times e^{-c \% F A T_{0}}$.

In Fig. 2, the fitted constant $c$ is given by $c=0.015$. It is shown that the 12 subjects (out of the 32 ) who completed all phases of the Minnesota Experiment (i.e., till the end of ad libitum refeeding) are a good representative subset of the 32 subjects that participated in the semistarvation (weight loss) phase and followed by restricted refeeding phase of the experiment, since the fitted curves are almost identical.

\section{Step 2. Temporal desynchronization in completion of fat and FFM recoveries}

For these 12 men of the Minnesota Experiment who completed the study, the relative changes in fat and FFM (in $\mathrm{kg}$ ) relative to the control (pre-starvation) period are shown during semistarvation (S12, S24) and refeeding (R12, R20) in Fig. 3 (left panel). The values shown are defined as $F A T_{\text {time }}-F A T_{\mathrm{C} 12}, F F M_{\text {time }}-F F M_{\mathrm{C} 12}$ and $W_{\text {time }}-W_{\mathrm{C} 12}$ for FAT, FFM and W, respectively. The data for FAT and FFM are corrected for changes in hydration and relative bone mass, using the correction factor $(\mathrm{k})$ determined in the Minnesota Experiment [35], are as follows: $F A T_{\text {corrected }}=k \times F A T_{\text {raw }}$, where $k=0.98,0.91,0.93,0.98$ at time C12, S24, R12 and $\mathrm{R} 20$, respectively, and $F F M_{\text {corrected }}=k \times F F M_{\text {raw }}$, where $k=0.88,0.92,0.99$ at time S24, R12 and R20, respectively; the raw and corrected data on body composition can be found as Supplementary Tables S1-S3. It is noticed that at R20,

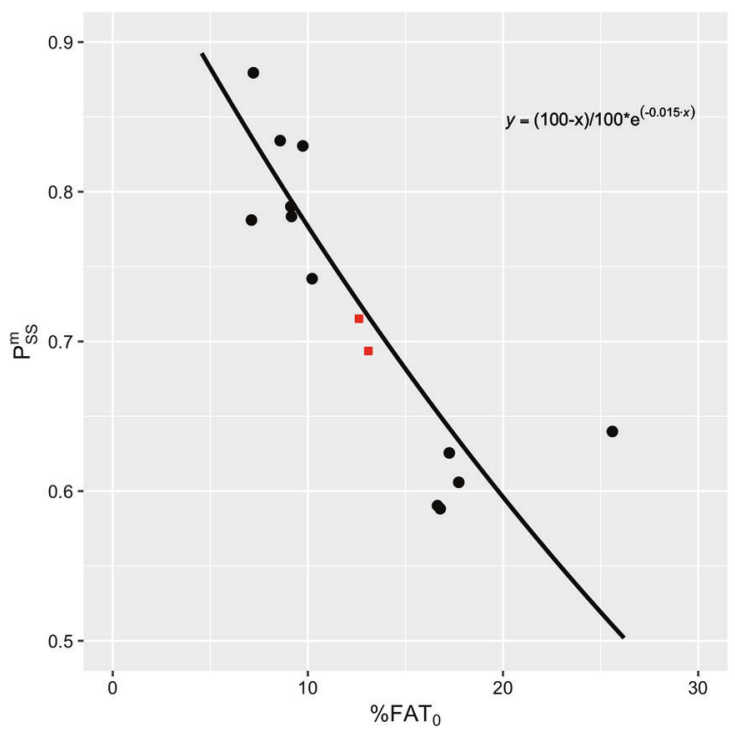

Fig. 2 Relationship between the semistarvation mass partition ratio $\left(P_{\mathrm{SS}}^{\mathrm{m}}\right)$ and the initial percentage body fat $\left(\% \boldsymbol{F A T} \boldsymbol{T}_{\mathbf{0}}\right)$. The expression $P_{\mathrm{SS}}^{\mathrm{m}}=\left(100-\% F A T_{0}\right) / 100 \times e^{-c \% F A T_{0}}$ given in equation 7 is used to fit the data. Left: Including only the 12 subjects that completed while all subjects have fully recovered or overshot their baseline (pre-starvation) body fat levels, with one exception they have not completed their FFM recovery. The interindividual variability for fat overshoot (range $0-9 \mathrm{~kg}$ ) and deficit in FFM $(-5$ to $0 \mathrm{~kg})$ are shown at time R20 in the right-hand panel of Fig. 3. The time-point, which is referred here as 'END', corresponds to an extrapolation of each subject's FFM at R20 to values corresponding to complete $(100 \%)$ recovery of their FFM; this calculation being made using the linear method for this extrapolation described in a section below.

\section{Step 3. Methods in estimating complete FFM recovery and accompanying fat overshoot}

In the analysis of fat overshooting, the following holds true: $\Delta W_{\mathrm{SS}}=W_{\mathrm{C} 12}-W_{\mathrm{S} 24}$ and $\Delta W_{\mathrm{RF}}=W_{\mathrm{END}}-W_{\mathrm{S} 24}$. By definition, $F F M_{\mathrm{END}}=F F M_{\mathrm{C} 12}$. To compute the value $F A T_{\mathrm{END}}$ at the final time END at which the subjects would have completely recovered their initial FFM, one may proceed as follows:

(I) Null method: setting $F A T_{\mathrm{END}}=F A T_{\mathrm{R} 20}$ although FFM is not completely recovered at time R20.

(II) Linear method: using a linear extrapolation in two steps: first we compute the time END through the equality

$F F M_{E N D}=F F M_{C 12}=F F M_{R 20}+$ Slope $_{F F M} \times(E N D-R 20)$,

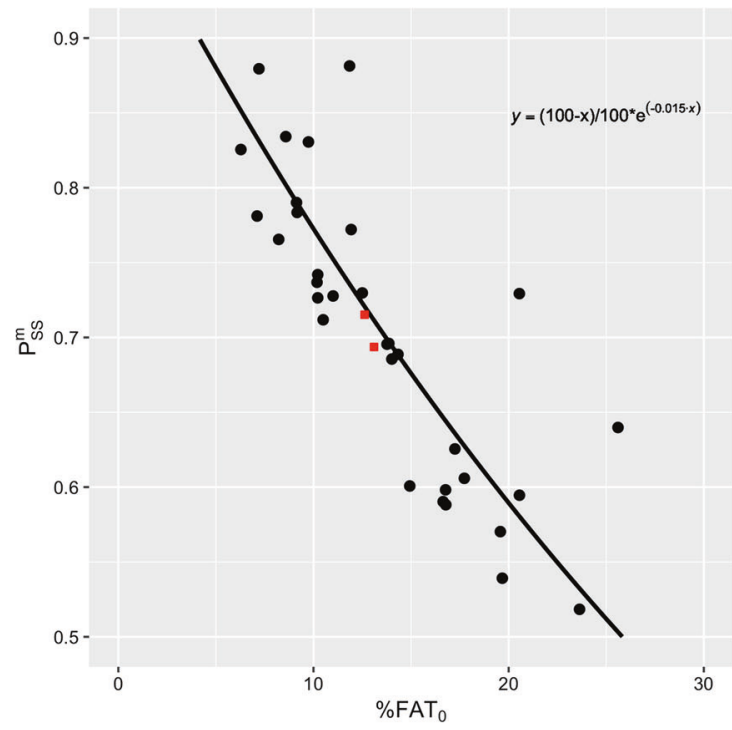

the whole Minnesota Experiment. Right: All the 32 subjects that participated to the Minnesota Experiment. The fitted constant is $c=0.015$. The two red squares correspond to the US Army Ranger data points presented in the section on 'Applications of the Model'. 

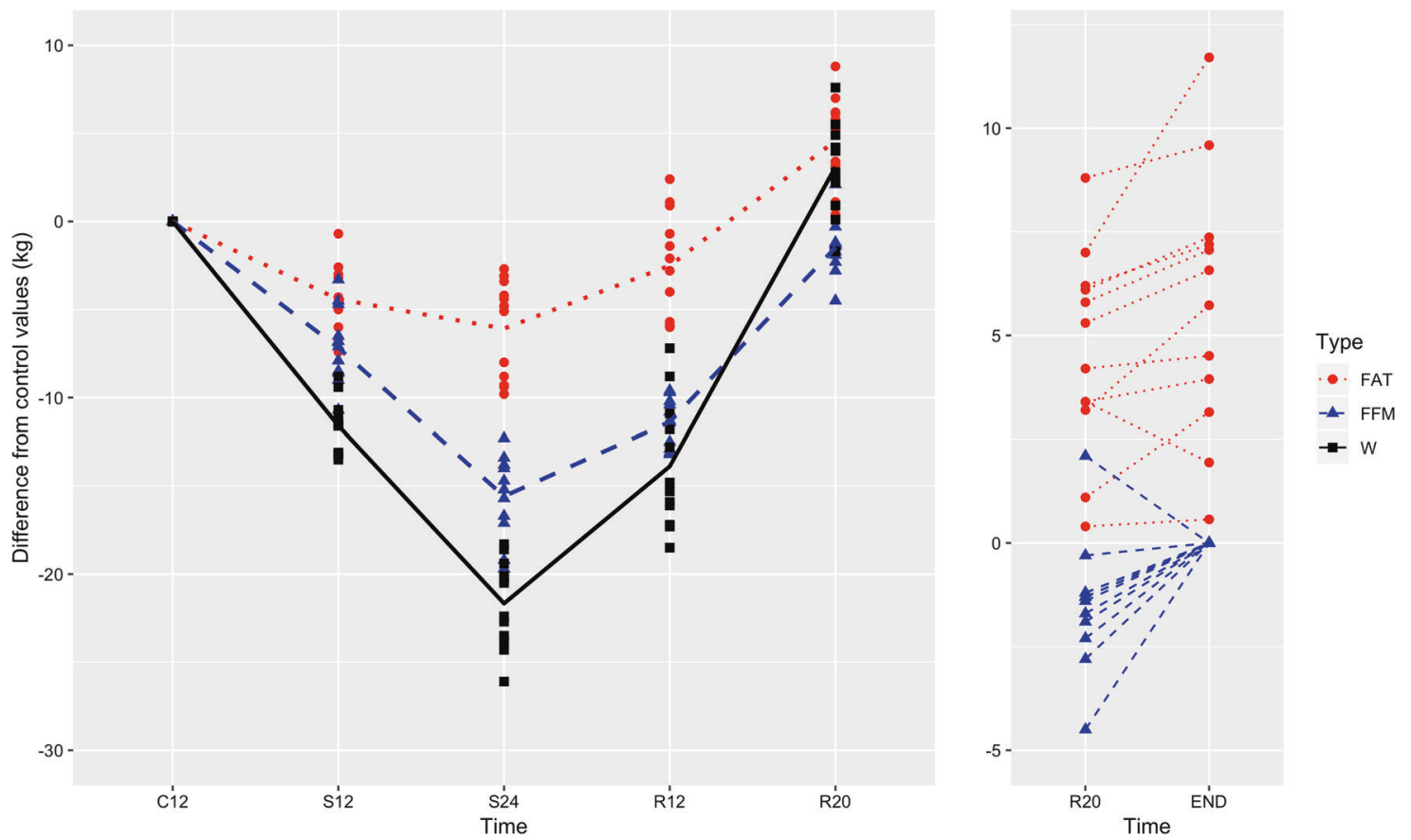

Fig. 3 Changes in fat and FFM relative to the control (prestarvation) period $(\mathrm{C12})$, at the end of the 24 weeks of semistarvation (S24) and at the end of refeeding phase (R20) in the 12 men who completed all phases of the Minnesota Experiment. All values are expressed as a difference from the corresponding values during the control (prestarvation) period. More precisely, the values are defined as $F A T_{\text {time }}-F A T_{\mathrm{C} 12}$ for FAT (red circle, dotted line),
$F F M_{\text {time }}-F F M_{\mathrm{C} 12}$ for FFM (blue triangle, dashed line) and $W_{\text {time }}-$ $W_{\mathrm{C} 12}$ for $\mathrm{W}$ (black square, solid line), for the 12 subjects that completed the Minnesota Experiment. As we shall see later, the final time points END are defined as the times at which the subjects would have completely recovered their initial FFM and are grouped here together for convenience. The lines are added to guide the eyes.

motivate us to model $(\gamma-1)$ as an exponential function of $\% F A T_{0}$ :

$$
\gamma-1=a e^{-b \% F A T_{0}}
$$

where the constants $a$ and $b$ are determined by fitting this model to the data.

Their exact values depend on the method used for computing the values $F A T_{\mathrm{END}}$ when the FFM has been completely recovered and on the type of statistical regression used. As explained previously, we consider four methods for computing $F A T_{\mathrm{END}}$. In Fig. 4, we show the fits obtained from a generalized linear model (GLM) with $95 \%$ confidence intervals (solid line) and from a linear model (LM), with $R^{2}$ values (dotted line). Following the performance of diagnostics tests to analyze the residuals, it is found that the GLM satisfies the major assumptions of regression analysis better than the LM, especially concerning the normality assumption of the error terms. Nevertheless, as shown in Fig. 4, both models give curves that are close to each other. In simple terms, the main difference between the above LM and GLM approaches to estimate the best parameter values for the constants $a$ and $b$ is the way the error term is handled. In the LM method, we write $y=a \times e^{b x} \times \varepsilon$, where $\varepsilon$ is a random error variable that follows a log-normal distribution with 

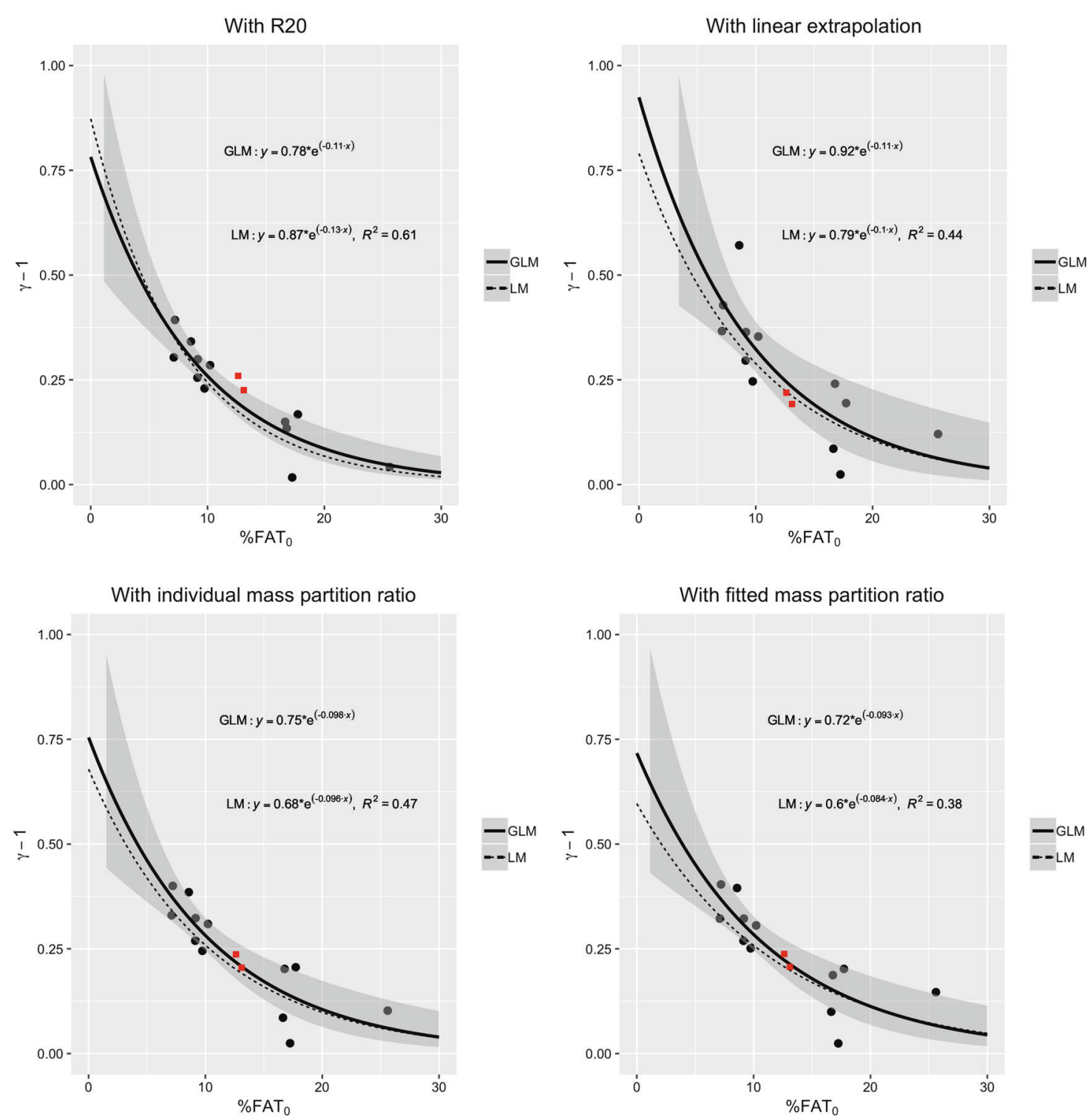

Fig. 4 The values for $(\gamma-1)$ are plotted vs. the initial fat percentage $\% \boldsymbol{F A T _ { 0 }}$. Two exponential fits are shown: a generalized linear model (GLM) with 95\% confidence intervals (solid line), and a linear model (LM), with $R^{2}$ values (dotted line). The four figures correspond to the four methods presented in the main text for computing the value

parameters $\mu=0$ and $\sigma^{2}$. Taking the logarithm on both sides of the equality gives $\log (y)=\log (a)+b x+\log (\varepsilon)$, which corresponds to the linear model $y^{\prime}=\beta_{0}+\beta_{1} x^{\prime}+\varepsilon^{\prime}$, where $y^{\prime}=\log (y), x^{\prime}=x, \beta_{0}=\log (a), \beta_{1}=\mathrm{b}$ and $\varepsilon^{\prime}=\log (\varepsilon)$ is a random error variable that follows a normal distribution with mean $\mu=0$ and variance $\sigma^{2}$. Note that in the LM method, the error term $\varepsilon$ is multiplied with the exponential function $y=a \times e^{b x}$. In the GLM method, the error term is instead added to the exponential function. Formally, we write $y=a \times e^{b x}+\varepsilon$, where $\varepsilon$ is a random error variable that follows a normal distribution with mean 0 and variance $\sigma^{2}$. As a consequence, the GLM admits the possibility of the value $y=0$ (which is excluded in LM) and assumes that the

$F A T_{\mathrm{END}}$ at the final time END at which the subjects would have completely recovered their initial FFM. The two red squares correspond to the US Army Ranger data points presented in the section on 'Applications of the Model'.

variance $V(y)$ is constant, while the LM method assumes that $V(y)$ varies with $x$. Finally, note that the LM method admits exact analytical solutions for the model parameters $a$ and $b$, while the GLM method requires numerical optimization algorithms to find the best values (the maximum likelihood estimates) for $a$ and $b$.

\section{Step 4. Relationship between $\gamma$ and $\% F A T_{0}$}

From equation 9, we have the approximation $\gamma-1=a e^{-b \% F A T_{0}}$, where the exact values of the fitted constants, $a$ and $b$, depend on the method used for computing the values for FAT when FFM has been completely 


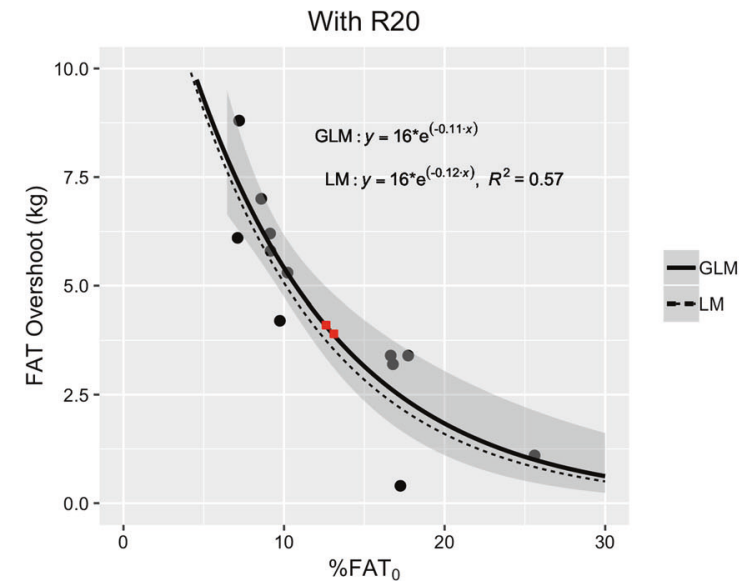

Fig. 5 Relationship between fat overshoot and initial adiposity (\% $F A T_{0}$ ), with fat overshoot expressed in absolute term (kg fat) in left panel, and expressed as a percentage of baseline body fat $\left(F A T_{0}\right)$ in

recovered. For example in Fig. 4, we observe that by using the linear extrapolation method and a generalized linear model, one obtains $a=0.92$ and $b=0.11$. In other words, the factor $\gamma$ may be well approximated with the following expression:

$\gamma=1+a e^{-b \% F A T_{0}}$.

\section{Model prediction of fat overshoot}

Combining this expression with equation 6 , one may predict the fat overshoot in a subject with initial fat percentage $\%$ $F A T_{0}$ and having lost weight $\Delta W_{\mathrm{SS}}$ through semistarvation:

FAT Overshoot $=a e^{-b \% F A T_{0}} \times \Delta W_{\mathrm{SS}}$.

It is to be noted that in the Minnesota Experiment, the percentage weight loss (25-29\%), as well as the absolute amount of weight loss $(18.3-26.1 \mathrm{~kg})$ were not too different among the subjects. In this case, with the assumption that $\Delta$ Wss is a constant, the relationship between FAT overshoot vs. $\% F A T_{0}$ can be plotted as shown in Fig. 5, rather than as FAT overshoot/ $\Delta$ Wss vs. $\% \mathrm{FAT}_{0}$ as we would expect from the model.

\section{Applications of the model}

\section{US Army Rangers fat overshooting}

As a first application, the model described here is used to make predictions for the fat overshoot in US Army Rangers who regained weight after 8 weeks of energy deficit resulting from intense training in a multistressor environment [49, 50], which will then be compared with actual observed (or measured) overshoot values. The data are presented as red square symbols in Figs. 2, 4, 5 and in Supplementary Table S3. We

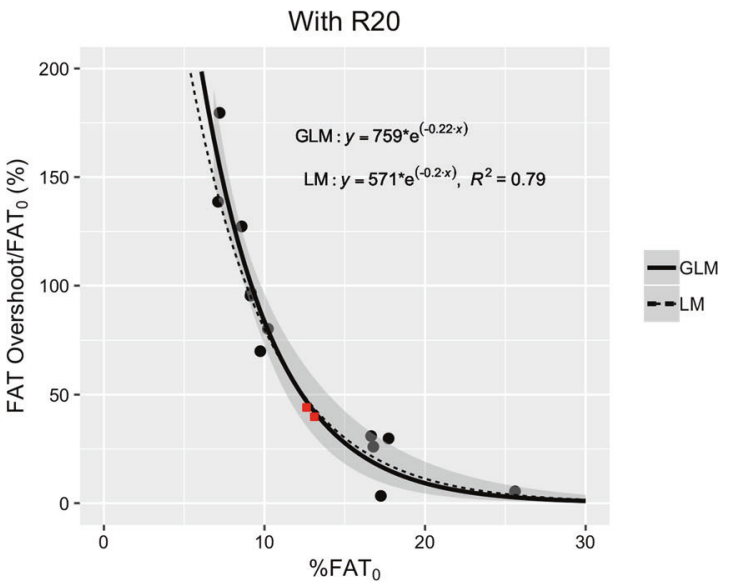

right panel. The two red squares correspond to the US Army Ranger data points presented in the section on 'Applications of the Model'.

have applied the same correction procedure as for the Minnesota data. In the publication of these studies [49, 50], only the mean values are accessible. The FAT overshoot is obtained by using the four methods presented previously to ensure that $F F M_{2}=F F M_{0}$; the data are provided in Supplementary Table S4. The predicted FAT overshoot is computed with equation 11 using the four methods and the GLM. As shown in Table S4, the predictions are very close to the observed values.

\section{Weight cycling from leanness to fatness}

The model is now used to predict body fat accumulation in three hypothetical subjects of identical weight $(70 \mathrm{~kg})$ but with different initial body fat $\%$ : 10\% (low), 20\% (medium) and $30 \%$ (high) who go through several successive weight cycles, with the lost and regain of $5 \mathrm{~kg}$ over each cycle (Fig. 6). To determine their weights at the end of the first cycle, we will use the equation 6 with the relation 10 for $\gamma$. To compute their weight at the end of the next cycles, we need to know how the values for $\gamma$ are updated at the end of each cycle. In other words, after the fat overshoot in one cycle, is the intrinsic leanfat partitioning over the next cycle the same as in the previous cycle or is it diminished as adiposity has increased, i.e., it moves to the right of the inverse exponential relating the leanfat partitioning to the initial adiposity [41, 42, 44]. This is unknown, and as it may depend on the timespan between two successive cycles, we shall consider two simple possibilities:

(i) The values for $\gamma$ are updated at each cycle according to the new body fat percentage and

(ii) The values for $\gamma$ are kept constant (the initial value of $\gamma$ corresponding to the initial fat percentage) at all cycles. For simplicity, the $\gamma$ values are obtained here by using the linear method for computing $F A T_{\mathrm{END}}$ and the GLM for the fit $(a=0.92, b=0.11)$. 


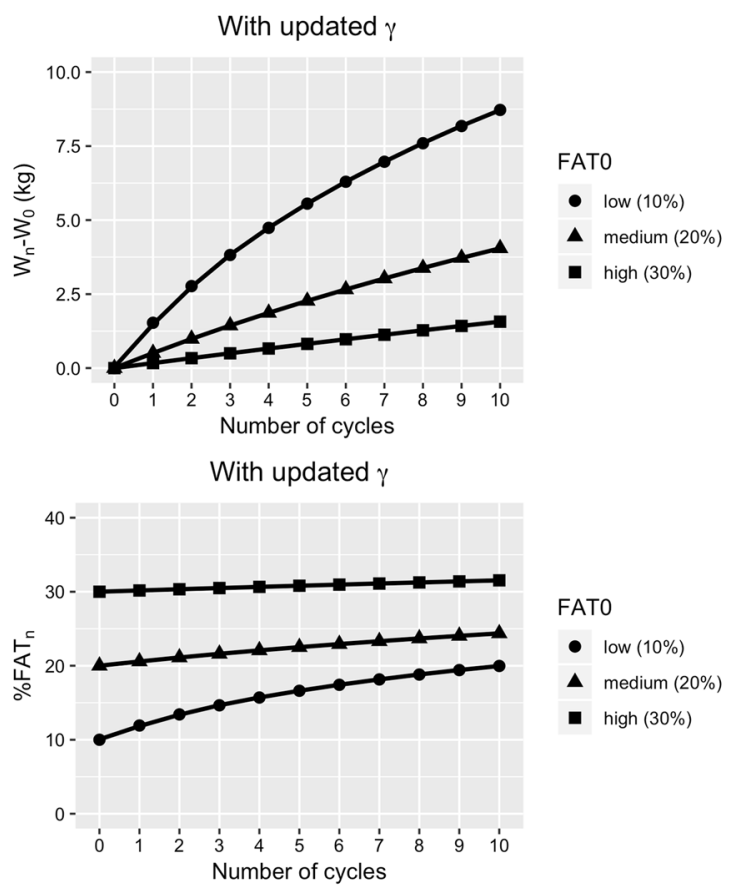

Fig. 6 Simulation of gain in body weight and body fat percentage in response to 10 weight cycles. Subjects of weight $70(\mathrm{~kg})$ and initial fat percentage $10 \%$ (low) or $20 \%$ (medium) or $30 \%$ (high) lose $5(\mathrm{~kg}$ ) at each cycle. We use the equations 6 and 10 and show the weight difference $W_{n}-W_{0}=F A T_{n}-F A T_{0}$, where $n=0, \ldots, 10$ is the number of cycles completed by the subjects. The $\gamma$ values are obtained by using the linear method for computing $F A T_{\mathrm{END}}$ and the GLM for

In the top panels of Fig. 6, we show the weight difference $W_{n}-W_{0}$ for 10 weight cycles. Note that since the FFM values are the same at the beginning and at the end of each cycle $\left(F F M_{n}=F F M_{0}\right)$, we actually have $W_{n}-W_{0}=$ $F A T_{n}-F A T_{0}$. On the left-hand side, we suppose that the values for $\gamma$ are updated at each cycle, according to the new body fat $\%$, leading to a decrease in fat overshoot at each new cycle. On the right-hand side, we assume that the values of $\gamma$ are all equal to the initial value of $\gamma$ corresponding to the initial fat $\%$, leading to a constant fat overshoot at each cycle and a linear increase in weight. In the bottom panels of Fig. 6, we show the body fat $\%$ of the subjects at each cycle. It is shown that the subject with the low initial fat\% (10\%) gets closer to the one with medium initial fat $\%(20 \%)$ as the number of cycles increases, and may even overtake him in the constant $\gamma$ situation. It can also be observed that the subject with high initial fat $\%$ (30\%) remains essentially at the same fat $\%$ during the 10 weight cycles; the predictions by our model of little or no fat overshooting in dieters with obesity is in accord with studies in which individuals with obesity when subjected to one cycle [51] or three successive cycles [52] of dieting failed to show altered body composition. More recent support for the notion that the parameter $\gamma$ is $\sim 1$ for people with very high initial body fat can also be derived from the
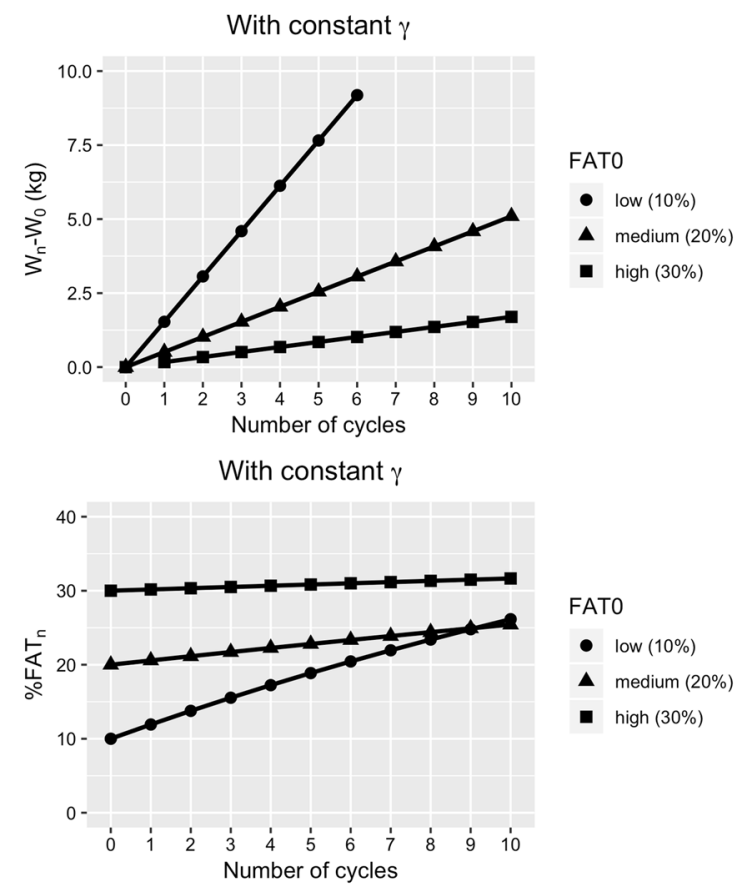

the fit $(a=0.92, b=0.11)$. Top left: the values of $\gamma$ are updated at each cycle, according to the new fat percentage, leading to a decrease in fat overshoot at each new cycle. Top right: the values of $\gamma$ are all equal to the initial value of $\gamma$ corresponding to the initial fat percentage, leading to a constant fat overshoot at each cycle and a linear increase in weight. Bottom panels: the fat percentage of the subjects at each cycle.

"Biggest Loser" study in which the subjects lost and regained body fat and FFM in the same proportion [53]. By contrast, even if the values for $\gamma$ are updated at each cycle in a lean dieter subjected to multiple weight cycles (with the amount of fat overshoot decreasing with each successive cycle), the cumulative amount of fat overshoot over several cycles will nonetheless result in the deposition of a substantial amount of excess of body fat.

In addition to uncertainties for updating of the values for $\gamma$ at each cycle, diet composition may also be a factor that can influence the asynchronous recovery of body fat and FFM and hence the factor $\gamma$. Indeed, an increased dietary fat intake was reported during weight recovery relative to baseline in the Army Ranger studies [49] and during the 'ad libitum refeeding' phase of the Minnesota Experiment [35]. Using a mathematical model of macronutrient balance, Hall [54] showed that the asynchronous recovery of body fat vs. FFM in the Minnesota Experiment may have been due to such changes in diet composition; furthermore, this model predicted recovery of the original body composition in the Minnesota men upon returning to the pre-starvation diet and physical activity, albeit after an extended duration ( $>1$ year) of consuming the baseline diet [54]. It should be noted, however, that excess dietary fat intake is unlikely to be the sole explanation for the asynchronous recovery of body fat 
vs. FFM in the Minnesota Experiment. Indeed, in the earlier phase of controlled refeeding lasting 12 weeks when dietary fat intake (in both absolute and relative terms) was actually lower than during the baseline period, a disproportionately faster recovery of body fat relative to FFM was also observed [35]; this was attributed to a sustained reduction of thermogenesis contributing to accelerated fat storage $[43,45]$. Overall, in our model presented here, it should be underlined that the parameter $\gamma$ may not only depend upon the time factor and the period of time between cycling pattern but also upon dietary composition.

\section{Concluding remarks}

While the prevalence of dieting to lose weight is more common in those people with obesity or overweight, it is substantial (and rising) in normal-weight population groups that include females and males, young and older adults, children and adolescents who perceive themselves as being too fat, as well as among athletes in weight-sensitive sports and among those in occupations where a slim image is professionally an advantage [34]. In these persons with initially normal weight, dieting attempts may predispose one to or represent another predisposition to future weight gain. Indeed, the loss of body weight has been shown to induce both metabolic and behavioral changes by which the body struggles to regain the weight $[55,56]$. In the context of dieting to lose weight, personal attitudes toward dieting, social pressure to diet or body image, as well as postslimming preoccupation with food, disinhibition and moral self-licensing for obesity-prone behavior may also act as a driver for weight regain, and contribute to fat overshooting. Obviously this mathematical model cannot account for all the determinants involved in fat overshooting, but represents merely the impact of an autoregulatory component and it has the merit to be simple and useful in practice, since it depends only on the measurable parameters $\% F A T_{0}$ and $\Delta W_{\text {SS }}$.

Acknowledgements This paper is dedicated to the late Jean Jacquet who played a pivotal role in the re-analysis of data from the Minnesota Experiment and their interpretation for novel concept development leading to mathematical modeling of human body composition regulation.

Funding This study was supported by intramural funding of the Department of Medicine, University of Fribourg, Switzerland.

Author contributions $\mathrm{PJ}$ and $\mathrm{AD}$ were involved in the study planning and data analysis. PJ performed the mathematical modeling, and AD, J-PM and YS contributed to data interpretation and application of the model. PJ and AD wrote the initial draft of the manuscript, and J-PM and YS contributed toward its final version. All authors read and approved the final version.

\section{Compliance with ethical standards}

Conflict of interest The authors declare that they have no conflict of interest.

Publisher's note Springer Nature remains neutral with regard to jurisdictional claims in published maps and institutional affiliations.

Open Access This article is licensed under a Creative Commons Attribution 4.0 International License, which permits use, sharing, adaptation, distribution and reproduction in any medium or format, as long as you give appropriate credit to the original author(s) and the source, provide a link to the Creative Commons license, and indicate if changes were made. The images or other third party material in this article are included in the article's Creative Commons license, unless indicated otherwise in a credit line to the material. If material is not included in the article's Creative Commons license and your intended use is not permitted by statutory regulation or exceeds the permitted use, you will need to obtain permission directly from the copyright holder. To view a copy of this license, visit http://creativecommons. org/licenses/by/4.0/.

\section{References}

1. Santos I, Sniehotta FF, Marques MM, Carraça EV, Teixeira PJ. Prevalence of personal weight control attempts in adults: a systematic review and meta-analysis. Obes Rev. 2017;18:32-50.

2. Mann T, Tomiyama AJ, Westling E, Lew AM, Samuels B, Chatman J. Medicare's search for effective obesity treatments: diets are not the answer. Am Psychol. 2007;62:220-33.

3. French SA, Jeffery RW, Forster JL, McGovern PG, Kelder SH, Baxter JE. Predictors of weight change over 2 years among a population of working adults; The Healthy Worker Project. Int J Obes. 1994;18:145-54.

4. Bild DE, Sholinsky P, Smith DE, Lewis CE, Hardin JM, Burke GL. Correlates and predictors of weight loss in young adults: the CARDIA study. Int J Obes. 1996;20:47-55.

5. Coakley EH, Rimm EB, Colditz G, Kawachi I, Willett W. Predictors of weight change in men: results from the Health Professionals Follow-up Study. Int J Obes. 1998;22:89-96.

6. Korkeila M, Rissanen A, Kaprio J, Sorensen TI, Koskenvuo M. Weight-loss attempts and risk of major weight gain: a prospective study in Finnish adults. Am J Clin Nutr. 1999;70:965-75.

7. Juhaeri J, Stevens J, Chambless LE, Tyroler HA, Harp J, Jones D. Weight change among self-reported dieters and non-dieters in white and African American men and women. Eur J Epidemiol. 2001;17:917-23.

8. Kroke A, Liese AD, Schulz M, Bergmann MM, KlipsteinGrobusch K, Hoffmann K, et al. Recent weight changes and weight cycling as predictors of subsequent two year weight change in a middle-aged cohort. Int J Obes. 2002;26:403-9.

9. Drapeau V, Provencher V, Lemieux S, Després JP, Bouchard C, Tremblay A. Do 6-y changes in eating behaviours predict changes in body weight? Results from the Quebec Family Study. Int J Obes. 2003;27:808-14.

10. Field AE, Aneja P, Austin SB, Shrier LA, de Moor C, GordonLarsen P. Race and gender differences in the association of dieting and gains in BMI among young adults. Obesity. 2007; $15: 456-64$

11. Sares-Jäske L, Knekt P, Männistö S, Lindfors O, Heliövaara M. Self-report dieting and long-term changes in body mass index and waist circumference. Obesity Sci Pract. 2019. Online version (preissue); https://onlinelibrary.wiley.com/doi/abs/10.1002/osp4.336. 
12. Stice E, Cameron RP, Killen JD, Hayward C, Taylor CB. Naturalistic weight-reduction efforts prospectively predict growth in relative weight and onset of obesity among female adolescents. J Consult Clin Psychol. 1999;67:967-74.

13. Viner RM, Cole TJ. Who changes body mass between adolescence and adulthood? Factors predicting change in BMI between 16 year and 30 years in the 1970 British Birth Cohort. Int J Obes. 2006;30:1368-74.

14. Neumark-Sztainer D, Wall M, Haines J, Story M, Eisenberg ME. Why does dieting predict weight gain in adolescents? Findings from project EAT-II: a 5-year longitudinal study. J Am Diet Assoc. 2007;107:448-55.

15. Neumark-Sztainer D, Wall M, Story M, Standish AR. Dieting and unhealthy weight control behaviors during adolescence: associations with 10-year changes in body mass index. J Adolesc Health. 2012;50:80-6.

16. Pietiläinen KH, Saarni SE, Kaprio J. Does dieting make you fat? A twin study. Int J Obes. 2012;36:456-64.

17. Field AE, Colditz GA. Frequent dieting and the development of obesity among children and adolescents. Nutrition. 2001;17:355-6.

18. Field AE, Austin SB, Taylor CB, Malspeis S, Rosner B, Rockett $\mathrm{HR}$, et al. Relation between dieting and weight change among preadolescents and adolescents. Pediatrics. 2003;112:900-6.

19. Balantekin KN, Savage JS, Marini ME, Birch LL. Parental encouragement of dieting promotes daughters' early dieting. Appetite. 2014;80:190-6.

20. Bacon L, Aphramor L. Weight science: evaluating the evidence for a paradigm shift. Nutr J. 2011;10:9.

21. Mann T, Tomiyama AJ, Ward A. Promoting public health in the context of the "obesity epidemic": false starts and promising new directions. Perspect Psychol Sci. 2015;10:706-10.

22. Bombak A, Monaghan LF, Rich E. Dietary approaches to weightloss, Health At Every Size ${ }^{\circledast}$ and beyond: rethinking the war on obesity. Soc Theory Health. 2019;17:89-108.

23. Cannon G, Einzig H. Dieting makes you fat. London: Century Publishing; 1983

24. Hill AJ. Does dieting make you fat. Br J Nutr. 2004;92:S15-S18.

25. Lowe MR, Timko CA. Dieting: really harmful, merely ineffective or actually helpful? Br J Nutr. 2004;92:S19-22.

26. Cannon G. Dieting. Makes you fat? Br J Nutr. 2005;93:569-70.

27. Lowe MR, Levine AS. Eating motives and the controversy over dieting: eating less than needed versus less than wanted. Obes Res. 2005;13:797-806.

28. Lowe MR. Dieting: proxy or cause of future weight gain? Obes Rev. 2015;16:19-24.

29. Bosy-Westphal A, Müller MJ. Measuring the impact of weight cycling on body composition: a methodological challenge. Curr Opin Clin Nutr Metab Care. 2014;17:396-400.

30. Bosy-Westphal A, Jäger J, Lagerpusch M, Skurk T, Müller MJ. Deep body composition phenotyping during weight cycling: relevance to metabolic efficiency and metabolic risks. Obes Rev. 2015;16:36-44.

31. Dulloo AG, Montani JP. Pathways from dieting to weight regain, to obesity and to the metabolic syndrome: an overview. Obes Rev. 2015;16:1-6.

32. National Task Force on the Prevention and Treatment of Obesity. Weight cycling. JAMA. 1994;272:1196-202.

33. Dulloo AG, Jacquet J, Montani JP. How dieting makes some fatter: from a perspective of human body composition autoregulation. Proc Nutr Soc. 2012;71:379-89.

34. Montani JP, Schutz Y, Dulloo AG. Dieting and weight cycling as risk factors for cardiometabolic diseases: who is really at risk? Obes Rev. 2015;16:7-18.

35. Keys A, Brozek J, Henschel A, Mickelsen O, Taylor HL. The biology of human starvation. Minnesota: University of Minnesota Press; 1950.
36. Dulloo AG, Jacquet J, Montani JP, Schutz Y. How dieting makes the lean fatter: from a perspective of body composition autoregulation through adipostats and proteinstats awaiting discovery. Obes Rev. 2015;16:25-35.

37. Payne PR, Dugdale AE. A model for the prediction of energy balance and body weight. Ann Hum Biol. 1977;4:525-35.

38. Christiansen E, Garby L. Prediction of body weight changes caused by changes in energy balance. Eur $\mathrm{J}$ Clin Invest. 2002;32:826-30.

39. Christiansen E, Garby L, Sørensen TI. Quantitative analysis of the energy requirements for development of obesity. J Theor Biol. 2005;234:99-106.

40. Caloin M. Modeling of lipid and protein depletion during total starvation. Am J Physiol Endocrinol Metab. 2004;287:E790-8.

41. Forbes GB. Lean body mass-body fat interrelationships in humans. Nutr Rev. 1987;45:225-31.

42. Hall KD. Body fat and fat-free mass inter-relationships: Forbes's theory revisited. Br J Nutr. 2007;97:1059-63.

43. Dulloo AG, Jacquet J, Girardier L. Autoregulation of body composition during weight recovery in human: the Minnesota experiment revisited. Int J Obes. 1996;20:393-405.

44. Dulloo AG, Jacquet J. The control of partitioning between protein and fat during human starvation: its internal determinants and biological significance. Br J Nutr. 1999;82:339-56.

45. Dulloo AG, Jacquet J. Adaptive reduction in basal metabolic rate in response to food deprivation in humans: a role for feedback signals from fat stores. Am J Clin Nutr. 1998; 68:599-606.

46. Dulloo AG, Jacquet J, Girardier L. Poststarvation hyperphagia and body fat overshooting in humans: a role for feedback signals from lean and fat tissues. Am J Clin Nutr. 1997;65:717-23.

47. Dulloo AG. Collateral fattening: when a deficit in lean body mass drives overeating. Obesity (Silver Spring). 2017;25:277-9.

48. Dulloo AG, Miles-Chan JL, Schutz Y. Collateral fattening in body composition autoregulation: its determinants and significance for obesity predisposition. Eur J Clin Nutr. 2018; 72:657-64.

49. Nindl BC, Friedl KE, Frykman PN, Marchitelli LJ, Shippee RL, Patton JF. Physical performance and metabolic recovery among lean, healthy men following a prolonged energy deficit. Int $\mathbf{J}$ Sports Med. 1997;18:317-24.

50. Friedl KE, Moore RJ, Hoyt RW, Marchitelli LJ, Martinez-Lopez LE, Askew EW. Endocrine markers of semistarvation in healthy lean men in a multistressor environment. J Appl Physiol. 2000;88:1820-30.

51. Wadden TA, Foster GD, Stunkard AJ, Conill AM. Effects of weight cycling on the resting energy expenditure and body composition of obese women. Int J Eat Disord. 1996;19:5-12.

52. Jebb SA, Goldberg GR, Coward WA, Murgatroyd PR, Prentice AM. Effects of weight cycling caused by intermittent dieting on metabolic rate and body composition in obese women. Int J Obes. 1991;15:367-74.

53. Fothergill E, Guo J, Howard L, Kerns JC, Knuth ND, Brychta $\mathrm{R}$, et al. Persistent metabolic adaptation 6 years after "The Biggest Loser" competition. Obesity (Silver Spring). 2016; 24:1612-9.

54. Hall KD. Computational model of in vivo human energy metabolism during semistarvation and refeeding. Am J Physiol Endocrinol Metab. 2006;291:E23-37.

55. MacLean PS, Blundell JE, Mennella JA, Batterham RL. Biological control of appetite: a daunting complexity. Obesity (Silver Spring). 2017;25:S8-S16.

56. Stubbs RJ, Duarte C, O’Driscoll R, Turicchi J, Michalowska J. Developing evidence-based behavioural strategies to overcome physiological resistance to weight loss in the general population. Proc Nutr Soc. 2019;78:576-89. 